\section{MS7-P8 New G-quadruplex DNA structures}

Boris L. Shivachev ${ }^{1}$, Louiza T. Dimowa ${ }^{2}$, Rosica P. Nikolova ${ }^{2}$, Hristina I. Sbirkova ${ }^{2}$, Lilia Tzvetanova ${ }^{2}$, Peter Hristoff ${ }^{1}$, Georgi Radoslavov $^{1}$, Doukov I. Tzanko ${ }^{3}$

1. Institute of Biodiversity and Ecosystem Research, Bulgarian Academy of Sciences 2 Gagarin Street, 1113 Sofia, Bulgaria

2. Institute of mineralogy and crystallography "Acad. Ivan Kostov"

3. Macromolecular Crystallographic Group, Stanford Synchrotron Radiation Lightsource, SLAC National Accelerator Laboratory, Stanford University, Stanford, CA 94309

email: blshivachev@gmail.com

The selection of potential new G-quadruplex DNA sequences has been performed using bioinformatics. The possible formation of G-quadruplex by the selected sequences has been assessed by PCR and fluorescence methods. Attempts for growing suitable single crystals of the selected DNA sequences has been performed in parallel. The results for G-quadruplex formation obtained by the molecular biology method and from crystallization results have been compared in order verify the viability of the methods.

Keywords: Nucleic acid, G-quadruplex, DNA

\section{MS7-P9 Radiation damage in protein-nucleic acid complexes}

Charles S. Bury ${ }^{1}$, John E. McGeehan ${ }^{2}$, Mikhail B. Shevtsov ${ }^{3}$, Alfred A. Antson ${ }^{4}$, Elspeth F. Garman ${ }^{1}$

1. Department of Biochemistry, University of Oxford, South Parks Road, Oxford, OX1 3QU

2. Biophysics Laboratories, Institute of Biomedical and Biomolecular Sciences, University of Portsmouth, King Henry I Street, Portsmouth, Hampshire PO1 2DY, UK

3. Laboratory of Structural Biology of GPCRs, Moscow Institute of Physics and Technology, Dolgoprudniy 141700, Russia

4. York Structural Biology Laboratory, Department of Chemistry, University of York, York Y010 5DD, UK

email: charles.bury@dtc.ox.ac.uk

Significant progress has been made over recent years in understanding how radiation damage mechanisms affect crystalline protein structure determination. Despite an active field studying the radiation chemistry of nucleic acids interacting with ionising radiation, few MX investigations exist on specific damage manifestations for crystalline DNA/RNA in their complexes with protein. Quantitative controlled comparisons between crystallised protein and nucleic acid damage mechanisms separately remain inherently difficult, but such challenges can be circumvented through investigating naturally forming nucleoprotein complexes. A recent study (1) utilised a model protein-DNA complex C.Esp1396I (2) to quantitatively investigate specific damage mechanisms for protein and DNA in a biologically relevant complex over a large dose range (2.07-44.63 MGy). A computational approach was developed to systematically locate damage sites, identifying typical specific damage sites on the complex. Strikingly the DNA component was determined to be far more resistant to specific damage than the protein for the investigated dose range.

For such complexes, the protein may be simply more susceptible to radiation damage, or may act as an electron/radical scavenger to protect DNA constituents. To address this issue, our previous computational strategy has been extended to statistically investigate damage dynamics in crystals of a large protein-RNA complex: TRAP (tryptophan-binding RNA attenuation protein) bound to 53 base RNA (3). The TRAP-RNA complex naturally crystallises in a 1:1 ratio with its RNA-unbound form, making it an ideal controlled experiment. RNA binding has been observed to stabilise susceptible protein residues, providing direct protection from electron density loss and disorder. Damage-susceptible acidic residues located far from the RNA-binding interface have increased decarboxylation rates upon RNA binding; the direct mechanisms behind this damage heterogeneity, and the implications of scavenging effects within crystalline nucleoprotein complexes are yet to be established.

\section{References}

1. Bury C, et al., McGeehan, JE (2015). J. Synchrotron Radiat. 22, 213-224

2. McGeehan JE, et al., Kneale GG (2008). Nucleic Acids Res. 36(14), 4778-87

3. Hopcroft NH, et al., Antson AA (2002). Nature 58(4), 615-621 